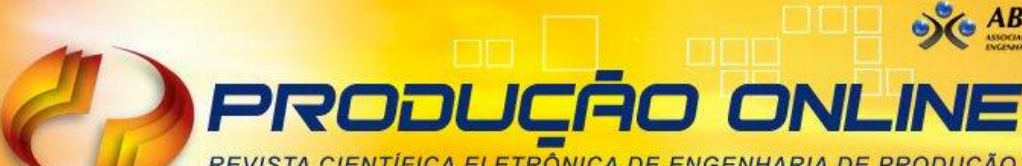 REVISTA CIENTIFICA ELETRÔNICA DE ENGENHARIA DE PRODUÇÃO

\section{SISTEMA DE APOIO À DECISÃO VIA PLANILHA ELETRÔNICA PARA O PLANEJAMENTO DE INTERCONEXÃO DE ERBS EM CENÁRIOS DE ALUGUEL COM DEMANDA E CUSTO FUZZY}

\section{DECISION SUPPORT SYSTEM BY WAY OF ELECTRONIC SCHEDULE FOR BTS INTERCONNECTION PLANNING ON RENTAL SCENARIOS WITH FUZZY DEMAND AND FUZZY COST}

\author{
Luttiely Santos de Oliveira* E-mail: luttiely@gmail.com \\ Marcos Antônio de Sousa*E-mail: masousa.damasio@gmail.com \\ Lorrane Cunha dos Santos* E-mail: lorrane.cunha10@gmail.com \\ Ricardo Luiz Machado* E-mail: drrmachado@gmail.com \\ *Pontifícia Universidade Católica de Goiás (PUC Goiás), Goiânia, GO
}

Resumo: A crescente demanda por novos serviços de transmissão de dados aliado as imprecisões de crescimento da demanda resultam na expansão dos Sistemas de Acesso Móvel Celular (SMC), requerendo intensa atividade de planejamento com uma grande variedade de opções técnicomercadológicas. Este artigo apresenta um Sistema de Apoio à Decisão para o dimensionamento da interconexão Estação Rádio Base - Central de Controle e Comutação em uma rede de telecomunicações móvel celular desenvolvido em planilha eletrônica. O principal objetivo é que o SAD possa auxiliar na escolha da topologia de rede mais viável economicamente, admitindo-se previsões imprecisas de demanda e custo e baseia-se no conceito de números fuzzy trapezoidais. A metodologia permite um alto grau de interatividade com o planejador, flexibilizando analisar cenários de contratos de aluguel para uma rede de pequeno porte, um método científico de investigação quantitativa. Os resultados mostram a eficiência da ferramenta computacional desenvolvida. A utilização do solver permitiu aprimorar o uso de uma ferramenta computacional de fácil utilização por parte do usuário final do sistema, pois o modelo matemático do Sistema de Apoio à Decisão utilizado demonstrou ser um facilitador para avaliar como é o comportamento da rede para as mais variadas possibilidades.

Palavras-chave: Sistema de Apoio à Decisão. Modelagem Matemática. Modelagem Fuzzy. Rede Móvel Celular. Planilha Eletrônica.

Abstract: The growing demand for new data transmission combined with the demand growth inaccuracies services results in the expansion of Mobile Access Systems, requiring intense planning activity with a variety of technical-marketing options. This paper presents a Decision Support System for the design of the Base Transceiver Station - Control and Switching Station interconnection in a mobile telecommunications network developed in a spreadsheet. The main objective is that the Decision Support System can help in choosing the most economically feasible network topology, assuming imprecise forecasts of demand and cost and based on the concept of trapezoidal fuzzy numbers. The methodology allows a high degree of interactivity with the planner, making it easier to analyze scenarios of rent contracts for a small network. The use of the solver allowed to improve the use of an easy-to-use computational tool by the end user of the system, since the mathematical model of the Decision Support System used proved to be a facilitator to evaluate how the network behavior for the most varied possibilities.

Keywords: Decision Support System. Mathematical Modeling. Fuzzy Modeling. Mobile Network. Electronic Spreadsheet. 


\section{INTRODUÇÃO}

A crescente demanda por novos serviços de transmissão de dados torna o setor de serviços em telecomunicações um mercado extremamente competitivo, em especial aquele voltado para prestação de serviços móveis. O aumento da diversidade de serviços aumenta também a indefinição sobre a quantidade de usuários que os serviços podem atingir, fazendo com que aquelas informações sobre demanda a ser atendida pelo sistema possuam um comportamento impreciso e difícil de serem estimadas durante a etapa de planejamento. Naturalmente, isto obriga as empresas operadoras a investirem continuamente tanto na evolução tecnológica quanto nos serviços oferecidos.

Consequentemente, a implantação e/ou expansão dos sistemas de telecomunicações requer intensa atividade de planejamento. Onde, quando, quanto e como investir, são questões para as quais o planejamento deve encontrar respostas. A enorme quantidade de opções técnico-mercadológicas a analisar requer de antemão escolhas por si mesmas difíceis. Além disso, o porte dos problemas e a velocidade das transformações exigem metodologias de planejamento consistentes, flexíveis e apoiadas em ferramentas computacionais. Os valores significativos geralmente envolvidos neste tipo de situação tornam desejável o uso de Sistemas de Apoio à Decisão (SAD), baseados em modelos matemáticos, como demonstrado em De Castro et al. (2016). Oliveira (2015), DeFigueiredo e CantoDosSantos (2013), Skianis (2013), Letournex et al. (2012), Rouskas et al. (2008), Allon e Federgruen (2007), Kasap et al. (2007), Niyato e Hossain (2006) e Anderson et al. (2006) são exemplos de trabalhos que utilizam modelos de otimização para avaliar o problema de alocação de recursos em redes de telecomunicação, com destaque para aquelas redes sem fio.

Diante deste cenário, as empresas operadoras do Sistema de Comunicação Móvel (SMC) também não podem deixar de avaliar a possibilidade de aluguel de parte da sua infraestrutura de rede de outra operadora de rede (INGA, AREVALO \& HINCAPIÉ, 2014). Neste cenário, informações como: a demanda de um determinado serviço, o custo de aluguel de um seguimento de rede, capacidade de atendimento de um enlace, são elementos fundamentais que precisam ser identificados pelo planejador. Porém, estas informações "precisas" nem sempre estão disponíveis 
durante a etapa de planejamento, o que é natural num contexto de rápido desenvolvimento tecnológico e surgimento de novos serviços.

Logo, uma característica bastante comum nas metodologias de planejamento é o fato de que o dimensionamento da rede acaba dependendo fortemente da precisão com que os dados de entrada são apresentados aos modelos, o que pode ser constatado no trabalho de DeSousa et al. (2013). A maneira de resolver o modelo matemático, não mais "exato", pode, portanto, mudar consideravelmente.

Neste trabalho é apresentado um modelo de Programação Linear Inteira Mista 0 - 1 (PLIM 0 - 1), utilizando a abordagem NÓ - ARCO (aplicação semelhante pode ser vista no estudo de Akasaka, D.'Agosto \& Ribeiro, 2016), para representar a rede de interconexão de ERBs. Manter o problema passível de solução, incorporando ao mesmo tempo aspectos da imprecisão, no sentido de flexibilizar o modelo e torná-lo mais fiel ao ambiente que pretende retratar, também é objetivo deste trabalho. $A$ imprecisão é incorporada através do uso de números fuzzy trapezoidais (George e Bo, 2008; Pedrycz e Gomide, 1998) para modelar os dados de custo e demanda. Esta modelagem matemática para os dados Fuzzy segue as orientações indicadas por DeSousa, Carlson \& Vieira (2015), Madronero et al. (2012) e Peidro et al. (2010), que utilizam esta técnica para resolver problemas de transporte com características similares àqueles que são avaliados neste artigo (SAM'AN et al., 2018, MATHUR, SRIVASTAVA \& PAUL, 2016).

Ainda, com o objetivo de fornecer uma ferramenta computacional de fácil manuseio por parte do usuário final, este trabalho também descreve o processo de programação via planilha eletrônica do Sistema de Apoio à Decisão proposto. $O$ SAD é implementado de modo a absorver as informações imprecisas existentes nos dados de custo de aluguel de rede e de demanda prevista para ser contratada em cada nó de Estação Rádio Base (ERB) do Sistema Móvel Celular. A modelagem de dados imprecisos (Fuzzy) apresentada aqui representa um avanço importante no trabalho desenvolvido em Oliveira e DeSousa (2015).

Considerando a conjuntura delineada, o objetivo é ter uma ferramenta para utilização de usuários pouco familiarizados com modelagem matemática e programação computacional para auxiliar na escolha da topologia de rede mais viável economicamente, a partir de dados relacionados a custos, demanda e capacidade técnica de equipamentos. As atividades mais importantes são: 
- Remodelar o problema tratado em Oliveira e DeSousa (2015) agregando a modelagem de dados fuzzy para as demandas previstas e custos de aluguel de rede. Como o software Excel não é uma ferramenta exclusiva de modelagem de problemas de otimização, o seu desempenho em termos de tempo computacional para resolver tais problemas é um pouco inferior àqueles utilizados em DeSousa et al. (2013);

- Implementação computacional, via planilha eletrônica, de um modelo de otimização que possa ser utilizado como Sistema de Apoio à Decisão no planejamento estratégico de interconexão de ERBs em um Sistema Móvel Celular. Cenários de contratos de aluguel de enlaces SDH são avaliados;

- Simulações de alguns cenários com redes de pequeno porte, com poucos nós de ERB e, consequentemente, com poucas variáveis de decisão (em torno de 70 variáveis).

Este artigo está organizado da seguinte forma: a seção 2 lista os elementos essenciais que compõem a infraestrutura de um sistema móvel celular; com destaque para a utilização da tecnologia SDH na interconexão de Estações Rádio Base; a seção 3 descreve a metodologia utilizada no desenvolvimento e implementação computacional do Sistema de Apoio à Decisão proposto; com destaque para a representação da rede, modelagem matemática e o tratamento dos dados imprecisos de demanda e custo; aplicações da ferramenta computacional desenvolvida podem ser conferidas nas seções 4 e 5; a seção 6 é reservada para as conclusões finais.

\section{INFRAESTRUTURA DE UM SISTEMA MÓVEL CELULAR}

De acordo com a Agência Nacional de Telecomunicações (ANATEL, 2014), o Serviço Móvel Celular (SMC) é o serviço de telecomunicações terrestre, que utiliza sistema de rádio comunicações que interconecta um terminal portátil particular à rede de telecomunicações.

\subsection{Descrição do Sistema Móvel Celular}

Uma determinada área de cobertura geográfica a ser atendida pelo serviço 
móvel celular é dividida em sub-regiões que são chamadas células (Figura 1), que é uma área atendida por uma Estação Rádio Base (ERB), dentro da qual a recepção do sinal atende as especificações do sistema.

Figura 1 - Principais componentes de um sistema de acesso móvel celular

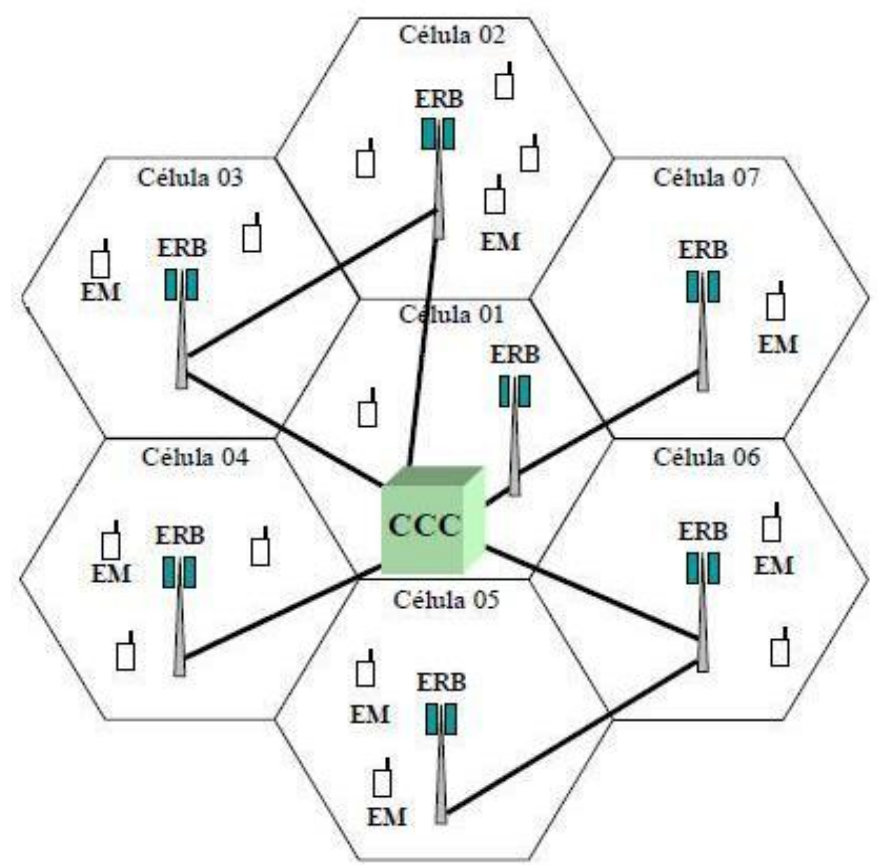

Fonte: DeSOUSA et al. (2013)

A Estação Rádio Base (ERB) aperfeiçoa a utilização do espectro de frequências disponíveis, sendo capaz de estabelecer comunicação com qualquer Estação Móvel (EM) que esteja em sua área de cobertura. É a ERB que faz a comunicação entre o assinante (interface rádio) e a Central de Comutação e Controle (CCC), em canais E1 de 2,048Mbp de taxa de transmissão. A disposição das ERBs em determinada área depende do tipo de interface rádio que é utilizada.

A Central de Comutação e Controle (CCC) é considerada o coração do sistema de acesso móvel celular. É o elemento que administra todas as Estações Rádio Base dentro de sua área de controle. Responsável pela validação dos assinantes, processamento de chamadas, estabelece a interface com a rede de comutação pública, interface com outras CCC's e gerenciamento de handoff (passagem do móvel de uma célula para outra). Informações mais detalhadas podem ser obtidas em Jeszensky (2004) ou Garg (2007). 


\subsection{Planejamento do sistema de acesso móvel celular}

No processo de planejamento de uma rede móvel celular, tem-se como objetivo obter a maior cobertura de atuação com a menor quantidade de equipamentos possível, e ao mesmo tempo prover a capacidade de rede necessária para se atender a demanda de tráfego dos usuários atendidos.

O transporte da informação é feito através de enlaces e nós de comunicações que são partes integrantes das redes. O planejamento tem a função principal de prover um meio eficaz no qual a organização possa atender suas necessidades futuras de conectividade, usando as redes que já existem como ponto de partida. Sendo o tempo um aspecto chave do planejamento de redes, pois à medida que o tempo passa, ocorrem novos eventos, e o planejamento é um processo ativo que faz uso contínuo das últimas inovações, fazendo regularmente um ajuste de planos.

Para facilitar o planejamento de um Sistema Móvel Celular, costuma ser realizado em três etapas:

- Comutação: responsável pelo dimensionamento da CCC - algumas respostas que esta etapa precisa apresentar: procedimentos de gerência, capacidade de atendimento de usuários, encaminhamento de solicitações de serviços, conexões com outros sistemas de telecomunicações, como, por exemplo, outras operadoras de SMC e operadoras de rede fixa;

- Radiofrequência: responsável pela definição da interface aérea, técnicas de modulação, localização de ERBs, potência de transmissão de cada ERB, área de cobertura, quantidade de canais necessários;

- Infraestrutura: escolhe a tecnologia e a capacidade dos equipamentos de transmissão a serem utilizados na interconexão das ERBs com a CCC. Esta etapa é responsável pelo dimensionamento da rede com o objetivo de escoar toda a demanda prevista para ser atendida nas ERBs até a CCC. A solução tecnológica mais utilizada para interconectar ERBs entre si e ERBs com a CCC em um sistema de comunicação móvel tem sido os sistemas SDH (Synchronous Digital Hierarchy) transmitido sobre fibra ótica. Outras soluções também podem ser encontradas no mercado, tais como: modems SHDSL (Symmetric high-speed digital subscriber line ou Linha Digital 
Simétrica de Assinante de Alta Velocidade) (Digitel, 2015) e rádio microondas (Ericsson, 2015).

O dimensionamento da infraestrutura é um processo que deve ser realizado de forma sequencial ao dimensionamento da radiofrequência. A modelagem matemática para o planejamento estratégico da infraestrutura de interconexão entre ERBs, e destas com a CCC, é um dos objetivos deste trabalho. O Sistema de Apoio à Decisão, implementado em planilha eletrônica, serve como ferramenta de auxílio para escolha da topologia de rede mais adequada para interligar as ERBs com CCC, utilizando a tecnologia SDH.

\subsection{SDH (Synchronous Digital Hierarchy - Hierarquia Digital Síncrona)}

A solução tecnológica mais utilizada para interconectar ERBs com ERBs e ERBs com a CCC em um Sistema Móvel Celular tem sido os sistemas SDH (Synchronous Digital Hierarchy) transmitindo sobre fibra ótica. Esta solução tecnológica permite maior largura de banda, ou seja, maior quantidade de informação por unidade de tempo.

O sistema SDH é um padrão internacional para redes ópticas de telecomunicações que especifica um sistema de transporte digital síncrono visando prover uma infra-estrutura de rede de telecomunicações mais simples, econômica e flexível.

Atualmente o padrão SDH utiliza frames STM-n com as seguintes taxas de bits: 155,520Mbit/s (STM-1 elétrico ou óptico), 622,080 Mbit/s (STM-4 óptico), 2.488,320 Mbit/s ou 2,5 Gbit/s (STM-16 óptico) e 9.953,280 Mbit/s ou $10 \mathrm{Gbit} / \mathrm{s}$ (STM-64 óptico), (TELECO, 2014).

De acordo com (TELECO, 2014), uma rede SDH é composta, basicamente, por:

- Rede física: que é o meio de transmissão que interliga os equipamentos SDH e pode ser composta por cabos de fibra óptica, enlaces de rádio e sistemas ópticos;

- Sistema de Gerência: que é o responsável pelo gerenciamento contendo as funcionalidades de supervisão e controle, e de configuração de equipamentos; 
- Equipamentos: são os multiplexadores SDH de diversas capacidades que executam o transporte de informações;

- Sistema de sincronismo: é o responsável pelo fornecimento das referências de relógio para os equipamentos e garante a propagação desse sinal pela rede.

\subsection{SDH na Interconexão de ERB-CCC}

A interconexão entre as ERBs e a CCC em um Sistema Móvel Celular é realizada através de links de canais E1, com taxa de transmissão de 2,048 Mbps. Estes links podem ser atendidos por enlaces SDH, com modulo de transmissão STM-1, que é capaz de agregar até 63 canais E1 de forma modular.

A Figura 2 mostra a utilização de sistemas SDH no processo de interconexão entre ERBs e a CCC, em Sistemas Móveis Celulares. Essa interconexão pode ser feita adotando ligações ponto a ponto, que representa o cenário mercadológico avaliado neste trabalho, onde a operadora do Sistema Móvel Celular pretende alugar links SDH para interligar as suas ERBs com a CCC. Existindo folga no seu sistema, a operadora de rede pode disponibilizar links para outras operadoras. Especificações técnicas mais detalhadas sobre os modems óticos SDH podem ser encontradas em Asga (2015).

A Figura 3 apresenta um exemplo de possível ocupação de um link SDH de capacidade modular de 63xE1. A operadora de rede utiliza 30 canais E1. Da folga disponível de 33xE1, ela aluga 24xE1 para duas operadoras de SMC, para que possam interligar os seus nós de ERB com as respectivas CCCs. 
Figura 2 - Interconexão ERBs - CCC utilizando tecnologia SDH

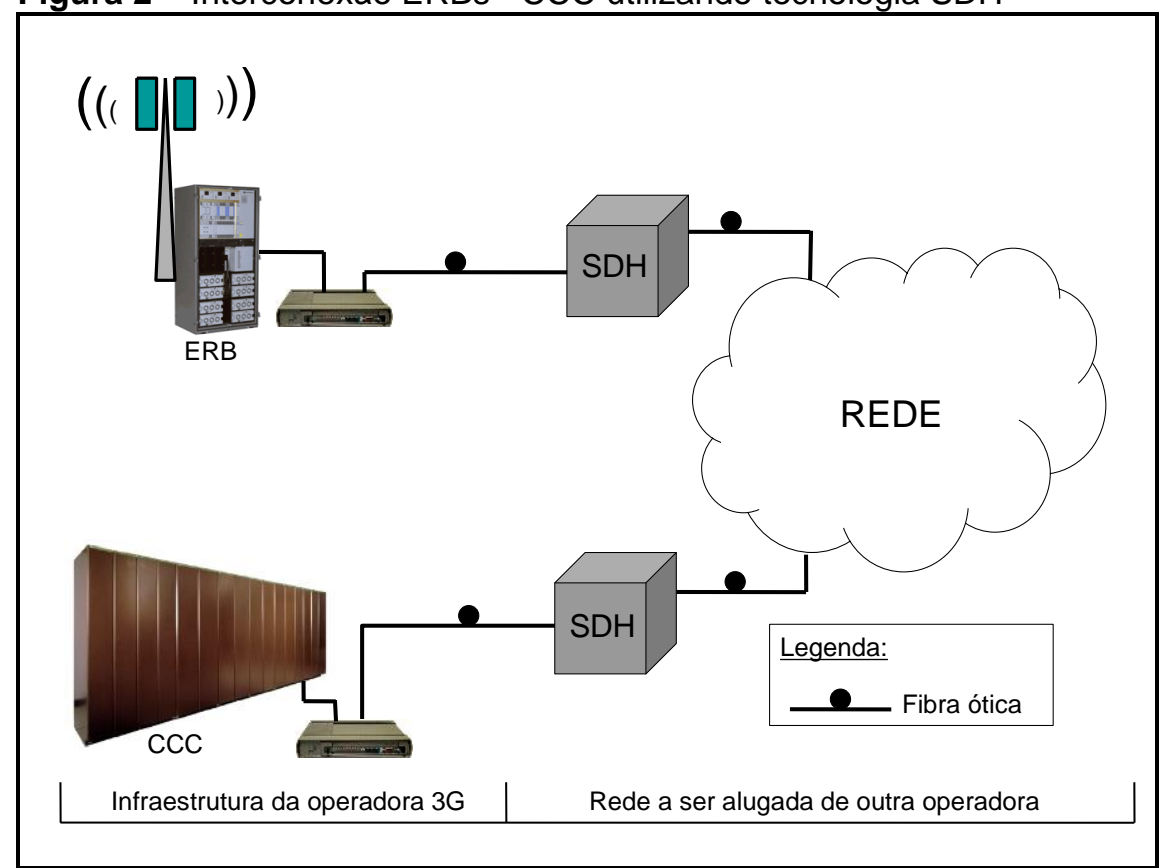

Fonte: DeSOUSA et al. (2013)

Figura 3 - Exemplo de compartilhamento de um link SDH

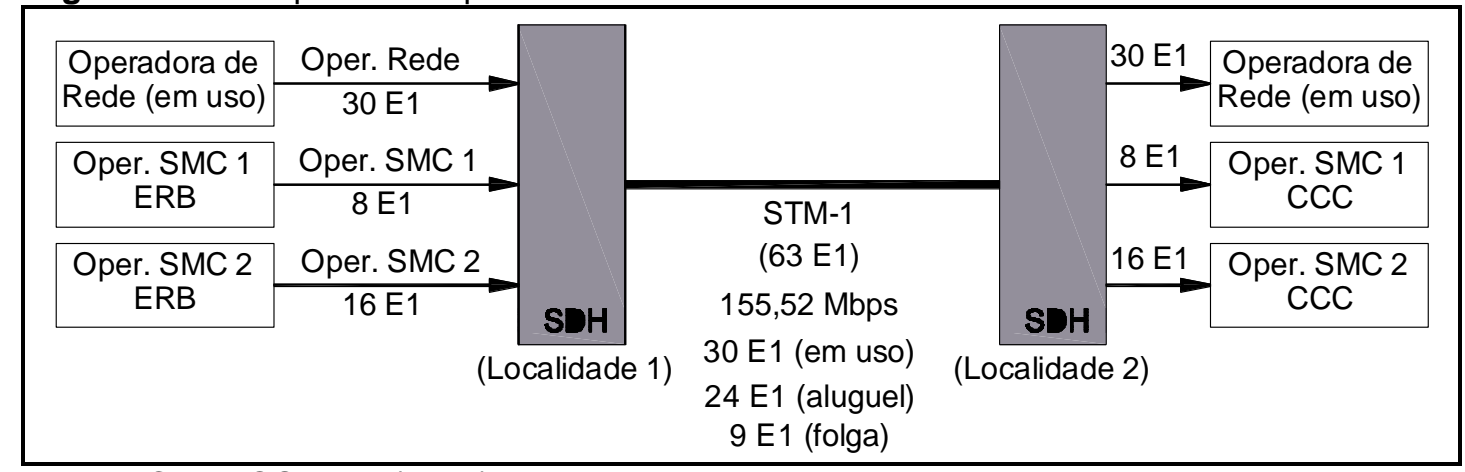

Fonte: CAMPOS et al. (2013)

\section{METODOLOGIA}

O problema tratado aqui teve como ponto de partida o trabalho de Oliveira e DeSousa (2015), onde a rede de interconexão ERB - CCC a modelagem segue a abordagem Nó - Arco. O modelo de Programação Linear Inteira Mista (PLIM 0-1) foi executado por um suplemento do Excel, a ferramenta solver, onde são configuradas as expressões matemáticas que representam as restrições de balanço de demanda, as restrições de capacidade técnica em cada arco previsto e a função objetivo de minimização de custo de aluguel de rede. 
Esse procedimento se assemelha ao método cientifico de investigação quantitativa, em delimitar o problema, realizar observações e interpretá-las com base nas relações encontradas, fundamentando-se nas teorias existentes.

\subsection{Representações da rede}

A rede candidata proposta para simulação do Sistema de Apoio à Decisão é composta por seis ERBs e uma CCC. As ERBs podem ser interconectadas com a CCC com enlaces diretos ou através de rotas alternativas (arcos candidatados para escoar o tráfego entre as ERBs). A Figura 4 mostra a rede indicando os arcos diretos (ERB - CCC) e indiretos (ERB - ERB) e o comprimento dos arcos, sendo 6 arcos diretos ERB - CCC e 12 arcos indiretos ERB - ERB.

Figura 4 - Rede Candidata

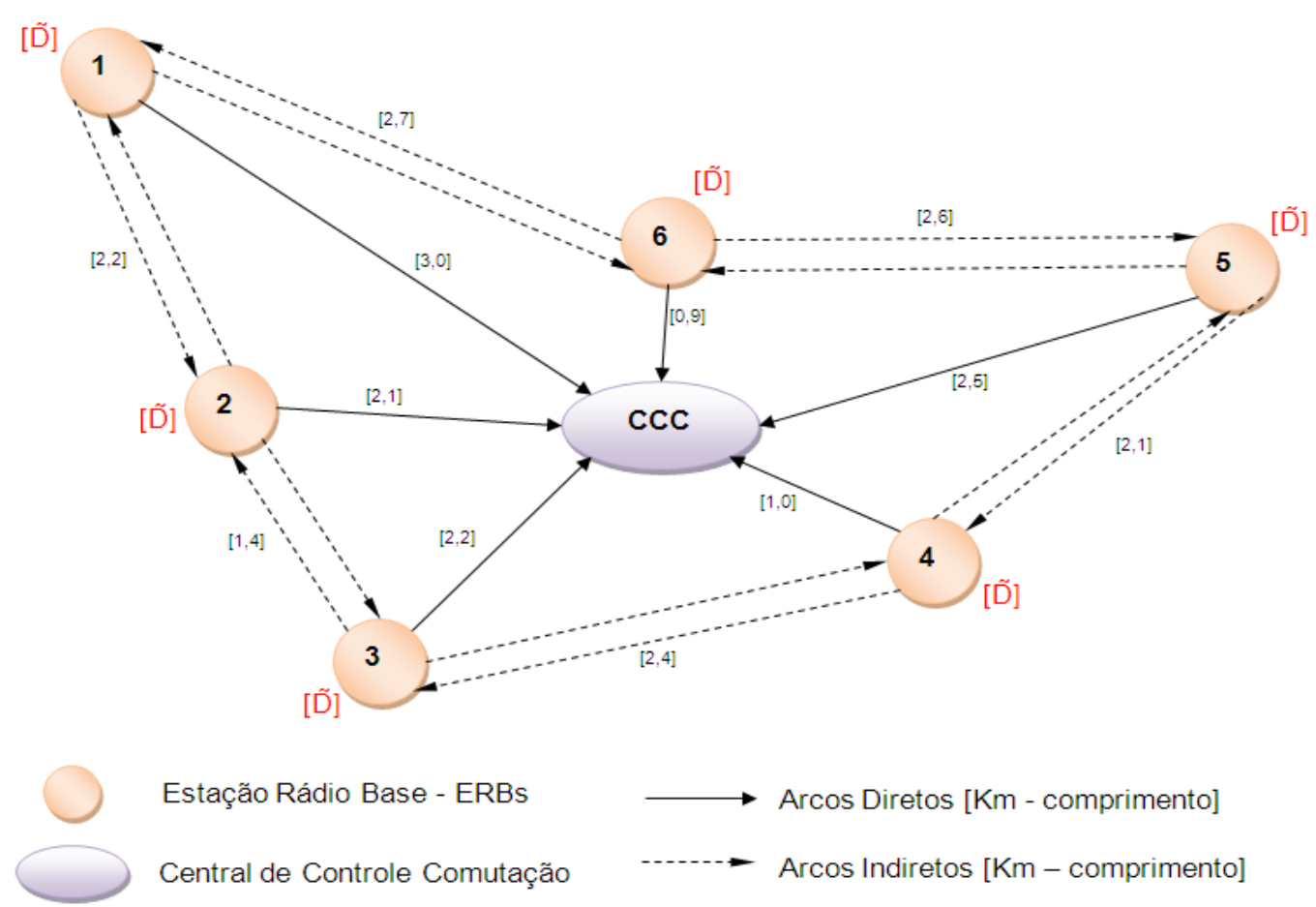

Fonte: elaborado pelos autores (2018)

Alguns parâmetros envolvidos no problema já são admitidos como sendo conhecidos e disponibilizados, como mostra na Tabela 1. Estes valores estão relacionados com a rede candidata ao qual foi montado o modelo que envolve a distância entre ERBs, a capacidade dos links SDH em canais E1, o custo de aluguel 
de rede óptica (por $\mathrm{km}$ ), o custo de aluguel de links SDH, o parâmetro de controle que mede a taxa de imprecisão da demanda e o intervalo da demanda, assim como seu valor crisp (exato).

Tabela 1 - Dados disponibilizados

Dados da Rede: Distância entre Nós e Demanda

\begin{tabular}{|c|c|c|c|c|c|c|c|}
\hline \multirow{2}{*}{$\begin{array}{l}\text { Nós de Origem } \\
\text { [i] }\end{array}$} & \multicolumn{7}{|c|}{$\begin{array}{l}\text { Nós de Destino [j] - Comprimento dos arcos diretos (BTS-BSC) e } \\
\text { indiretos (BTS-BTS) em Km }\end{array}$} \\
\hline & $\begin{array}{c}\text { CCC } \\
01\end{array}$ & ERB 01 & ERB 02 & ERB 03 & ERB 04 & ERB 05 & ERB 06 \\
\hline ERB 01 & 3,0 & & 2,2 & & & & 2,7 \\
\hline ERB 02 & 2,1 & 2,2 & & 1,4 & & & \\
\hline ERB 03 & 2,2 & & 1,4 & & 2,4 & & \\
\hline ERB 04 & 1,0 & & & 2,4 & & 2,1 & \\
\hline ERB 05 & 2,5 & & & & 2,1 & & 2,6 \\
\hline ERB 06 & 0,9 & 2,7 & & & & 2,6 & \\
\hline
\end{tabular}

Dados da Tecnologai SDH - Capacidade de Link e Custo de Aluguel :

Link SDH

Link21 Link42 Link63

Capacidade Link SDH

Valor Médio custos

\begin{tabular}{ccc}
21 & 42 & 63 \\
\hline 1,0 & 1,7 & 2,5 \\
\hline
\end{tabular}

Dados de Rede: Custo/Km de aluguel de Rede Óptica :

0,10

Alfa $(\alpha)$ : Fator de confiança $[0,1]$

0,0

Valores de demanda para alfa atual

\begin{tabular}{cccc} 
& $\begin{array}{c}\text { Valor crisp } \\
\text { demanda }\end{array}$ & Demanda mínima & Demanda máxima \\
\cline { 2 - 4 } ERB 01 & 44 & 40 & 48 \\
\hline ERB 02 & 30 & 27 & 33 \\
\hline ERB 03 & 38 & 35 & 41 \\
\hline ERB 04 & 14 & 12 & 16 \\
\hline ERB 05 & 34 & 31 & 37 \\
\hline ERB 06 & 20 & 18 & 22 \\
\hline
\end{tabular}

Fonte: Autor

Revista Produção Online. Florianópolis, SC, v. 18, n. 4, p. 1227-1253, 2018. 
Esses parâmetros são de fundamental importância, pois é a partir deles que é estruturado o modelo matemático, de forma a atender e satisfazer todas as equações, analisando todos os pontos sem diminuir variáveis ou analisar células de variáveis que não estão dentro da rede proposta, fazendo com que o tempo computacional para otimizar o modelo seja maior que o necessário.

\subsection{Representações dos dados imprecisos}

Tanto o custo de aluguel de link quanto à demanda a ser atendida em cada ERB são parâmetros difíceis de estimar com precisão por serem influenciados por fatores como flutuação de preços de mercado, surgimento de novas tecnologias, situação político-econômica, entre outros. O dimensionamento da rede depende destas informações. Assim, optou-se por modelar estes parâmetros utilizando números fuzzy, que abrange uma faixa de valores possíveis resultando em simulações mais realísticas e com maior flexibilidade.

A cada uma das demandas das ERBs $\left(\tilde{D}_{k}\right)$ e, também, dos custos de link $\operatorname{SDH}\left(\tilde{C}_{n}\right)$ foi associado um número fuzzy: $\tilde{D}_{k}=\left(d_{a}, d_{b}, d_{c}, d_{d}\right)$ e $\tilde{C}_{n}=\left(c_{a}, c_{b}, c_{c}, c_{d}\right)$, que define o intervalo de variação do dado. Empregou-se uma função de pertinência trapezoidal simétrica, conforme Figura 5. O valor médio (VM) representa o valor de maior pertinência.

Figura 5 - Função de Pertinência: Modelagem Trapezoidal

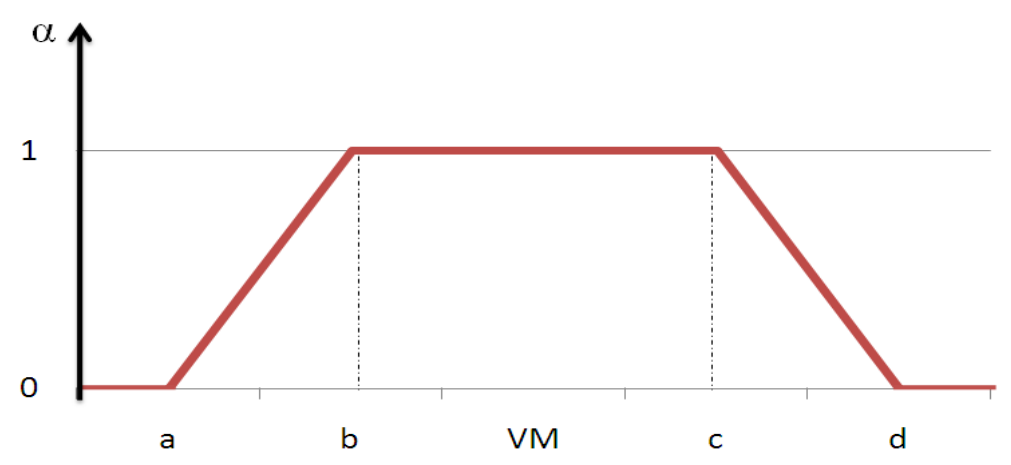

Fonte: elaborado pelos autores (2018)

\subsection{Formulações matemática do SAD}

As variáveis de decisão do modelo se referem à: 
Valor de fluxo de demanda escoado pelo arco: representado por Yij, onde $Y$ indica a quantidade de canais E1 escoada pelo arco que liga a ERB [i] à ERB [j] ou à CCC [j]. A Tabela 2 indica as células da planilha utilizadas para representar estas variáveis reais. Pode-se observar que para o cenário avaliado existem 18 variáveis Yij.

Tabela 2 - Fluxo de Demanda: Varáveis reais, utilizadas para se obter o fluxo de demanda escoado pelos arcos (links) da rede

\begin{tabular}{|c|c|c|c|c|c|c|c|}
\hline \multirow{2}{*}{$\begin{array}{l}\text { Nós de } \\
\text { Origem } \\
\text { [i] }\end{array}$} & \multicolumn{7}{|c|}{ Nós de Destino [j] - Variáveis de Fluxo Yij (em Canais E1) - Reais } \\
\hline & \multirow[t]{2}{*}{$\operatorname{CCC} 01$} & \multirow[t]{2}{*}{ ERB 01} & \multirow[t]{2}{*}{ ERB 02} & \multirow[t]{2}{*}{ ERB 03} & \multirow[t]{2}{*}{ ERB 04} & \multirow[t]{2}{*}{ ERB 05} & \multirow[t]{2}{*}{ ERB 06} \\
\hline ERB 01 & & & & & & & \\
\hline ERB 02 & & & & & & & \\
\hline ERB 03 & & & & & & & \\
\hline ERB 04 & & & & & & & \\
\hline ERB 05 & & & & & & & \\
\hline ERB 06 & & & & & & & \\
\hline
\end{tabular}

Fonte: elaborado pelos autores (2018)

Escolha (ou não) do link para o escoamento da demanda prevista: representada por Xijn, onde o valor de X (0 ou 1), indica se o link SDH de capacidade [n] é ou não alocado no arco que liga a ERB [i] à ERB [j] ou à CCC [j]. A Tabela 3 indica a planilha utilizada para representar estas variáveis binárias. Podese observar que para o cenário avaliado existem 54 variáveis Xijn. 
Tabela 3 - Escolha de Link SDH: Varáveis Binárias, utilizadas para se escolher (ou não) um link SDH

\begin{tabular}{|c|c|c|c|c|c|c|c|c|c|c|c|c|c|c|c|c|c|c|c|c|c|c|}
\hline \multicolumn{23}{|c|}{ Nós de Destino [j]) - Variáveis de Alocação de Link SDH Xijn - Binárias } \\
\hline \multirow{3}{*}{$\begin{array}{l}\text { Nós de } \\
\text { Origem } \\
\text { [i] }\end{array}$} & \multirow{2}{*}{$\begin{array}{l}\text { Capacidade } \\
\text { Link SDH }\end{array}$} & \multicolumn{3}{|c|}{$\operatorname{ccC} 01$} & \multicolumn{3}{|c|}{ ERB 01} & \multicolumn{3}{|c|}{ ERB 02} & \multicolumn{3}{|c|}{ ERB 03} & \multicolumn{3}{|c|}{ ERB 04} & \multicolumn{3}{|c|}{ ERB 05} & \multicolumn{3}{|c|}{ ERB 06} \\
\hline & & 21 & 42 & 63 & 21 & 42 & 63 & 21 & 42 & 63 & 21 & 42 & 63 & 21 & 42 & 63 & 21 & 42 & 63 & 21 & 42 & 63 \\
\hline & $\begin{array}{c}\text { Custo Link } \\
\text { SDH }\end{array}$ & 1,0 & 1,7 & 2,5 & 1,0 & 1,7 & 2,5 & 1,0 & 1,7 & 2,5 & 1,0 & 1,7 & 2,5 & 1,0 & 1,7 & 2,5 & 1,0 & 1,7 & 2,5 & 1,0 & 1,7 & 2,5 \\
\hline $\begin{array}{c}\text { ERB } \\
01\end{array}$ & & & & & & & & & & & & & & & & & & & & & & \\
\hline $\begin{array}{c}\text { ERB } \\
02\end{array}$ & & & & & & & & & & & & & & & & & & & & & & \\
\hline $\begin{array}{c}\text { ERB } \\
03\end{array}$ & & & & & & & & & & & & & & & & & & & & & & \\
\hline $\begin{array}{c}\text { ERB } \\
04\end{array}$ & & & & & & & & & & & & & & & & & & & & & & \\
\hline $\begin{array}{c}\text { ERB } \\
05\end{array}$ & & & & & & & & & & & & & & & & & & & & & & \\
\hline $\begin{array}{c}\text { ERB } \\
06\end{array}$ & & & & & & & & & & & & & & & & & & & & & & \\
\hline
\end{tabular}

Fonte: elaborado pelos autores (2018)

A presença de números fuzzy nas restrições do modelo PLIM pode alterar substancialmente o procedimento de resolução. Há a necessidade de se transformar os números fuzzy de maneira a permitir o seu tratamento. Este processo é denominado "defuzzyficação". Existem várias técnicas para defuzzyficação (Pedrycz e Gomide, 1998). Contudo, neste trabalho, para os dados de demanda, optou-se por substituir o número fuzzy por um valor que permita resolver o problema de forma mais simples sem perder as características de imprecisão do número fuzzy. $\mathrm{O}$ método consiste em obter um número crisp a partir de uma função de parametrização, na qual um fator de confiança, $\alpha \in[0,1]$, expressa o quão preciso são os dados de demanda. Maiores detalhes destas abordagens podem ser encontrados em Madronero et al. (2012) e Peidro et al. (2010).

As restrições do modelo de otimização são reunidas em dois conjuntos:

Conjunto 1: Restrições de Balanço de Demanda que garantem o balanço de fluxo de demanda em todos os nós de ERB. A expressão 1 utilizada para fazer este balanço em cada ERB da rede tratada no Oliveira e DeSousa (2015), foi modificada para atender a imprecisão com a expressão 2 e 3 . Onde a expressão 2 representa o limite inferior para a demanda prevista para ser atendida e a expressão 3 o limite superior desta mesma demanda prevista,. Este controle é realizado conforme o 
grau de confiança, $\alpha$, que o planejador aponta para as demandas previstas em cada ERB.

$$
\begin{aligned}
& \sum_{[k, i] \in A_{\theta}} Y_{k i}-\sum_{[j, k] \in A_{\theta}} Y_{j k}=d_{k} \forall k \in O_{E R B} \\
& \sum_{[k, j] \in A_{m}} Y_{k i}-\sum_{[j, k] \in A_{m}} Y_{j k} \geq \frac{\alpha}{2} \cdot \frac{d_{k e}+d_{k d}}{2}+\left(1-\frac{\alpha}{2}\right) \cdot \frac{d_{k a}+d_{k b}}{2} \forall k \in O_{E R B} \\
& \sum_{[k, i] \in A_{m}} Y_{k i}-\sum_{[j, k,] \in A_{i n}} Y_{j k} \leq \frac{a}{2} \cdot \frac{d_{k a}+d_{k b}}{2}+\left(1-\frac{a}{2}\right) \cdot \frac{d_{k v}+d_{k d}}{2} \forall k \in O_{E R B}
\end{aligned}
$$

Onde:

Ae: conjunto de arcos de escoamento de demanda (diretos + indiretos), que ligam os nós de ERB [i] aos nós de ERB [j] ou ao nó de CCC [j];

OERB: conjunto de todos os nós de ERB da rede;

Yki: variável real que representa o fluxo de demanda atendido pelo arco [k, i] $\epsilon$ Ae, com a demanda saindo do nó [k] e chegando em [i];

Yjk: variável real que representa o fluxo de demanda atendido pelo arco [j, k] $\epsilon$ Ae, com a demanda saindo do nó [j] e chegando em [k];

Dk: demanda prevista, em canais $\mathrm{E} 1$, para ser atendida em cada nó [k] $\epsilon$ OERB.

Conforme a Tabela 4, as restrições 2 e 3 balanceiam os fluxos mínimos de demanda (limite inferior) e máximos de demanda (limite superior).

Tabela 4 - Restrições de Balanço de Demanda em cada ERB da Rede

Limite inferior

\begin{tabular}{cccc}
$\begin{array}{c}\text { Conjunto de Nós } \\
\text { de ERB da Rede }\end{array}$ & $\begin{array}{c}\text { Fluxo } \\
\text { Entr. }\end{array}$ & $\begin{array}{c}\text { Fluxo } \\
\text { Saída }\end{array}$ & $\begin{array}{c}\text { Fluxo } \\
\text { Rede }\end{array}$ \\
\hline ERB 01 & 40 & 0 & -40 \\
ERB 02 & 27 & 0 & -27 \\
ERB 03 & 35 & 0 & -35 \\
ERB 04 & 12 & 0 & -12 \\
ERB 05 & 31 & 0 & -31 \\
ERB 06 & 18 & 0 & -18
\end{tabular}

Limite superior

\begin{tabular}{cccc}
\hline $\begin{array}{c}\text { Conjunto de Nós } \\
\text { de ERB da Rede }\end{array}$ & $\begin{array}{c}\text { Fluxo } \\
\text { Entr. }\end{array}$ & $\begin{array}{c}\text { Fluxo } \\
\text { Saída }\end{array}$ & $\begin{array}{c}\text { Fluxo } \\
\text { Rede }\end{array}$ \\
\hline ERB 01 & 48 & 0 & -48 \\
ERB 02 & 33 & 0 & -33 \\
ERB 03 & 41 & 0 & -41 \\
ERB 04 & 16 & 0 & -16 \\
ERB 05 & 37 & 0 & -37 \\
ERB 06 & 22 & 0 & -22
\end{tabular}

Fonte: elaborado pelos autores (2018) 
Conjunto 2: Restrições de Capacidade ocorrem em cada arco previsto para escoar a demanda das ERBs. Estas restrições asseguram que a capacidade do link SDH escolhido seja capaz de atender a demanda escoada pelo arco. Representada pela inequação (4) utilizada para fazer este balanço em cada arco previsto para a rede.

$$
\sum_{[n] \in O_{S N H}} \operatorname{Cap}_{i j}^{n} \cdot X_{i j n} \geq Y_{i j} \forall\left[i_{j} j\right] \in A_{B}
$$

Onde:

OSDH: conjunto de capacidades dos links SDH candidato no arco $[i, j]{ }^{\epsilon} \mathrm{Ae}$; $\operatorname{Cap}_{i j:}^{n}$ : capacidade do link SDH de modularidade [n] candidato no [i, j] ${ }^{E} \mathrm{Ae}$;

Yij: variável real que representa o fluxo de demanda atendido pelo arco [i, j] $\epsilon$ Ae;

Xijn: variável binária que representa a escolha do link SDH de capacidade [n] candidato no arco $[\mathrm{i}, \mathrm{j}]{ }^{\epsilon} \mathrm{Ae}$.

$\mathrm{Na}$ Tabela 5 são indicados na planilha as modularidades escolhidas em cada enlace (21xE1, 42xE1 ou 63xE1), bem como a demanda escoada pelo mesmo e a folga de rede. 


\begin{tabular}{|c|c|c|c|}
\hline $\begin{array}{l}\text { Conjunto de Arcos Diretos e } \\
\text { Indiretos da Rede }\end{array}$ & $\begin{array}{l}\text { Cap. } \\
\text { SDH }\end{array}$ & $\begin{array}{l}\text { Dem. } \\
\text { Link }\end{array}$ & $\begin{array}{l}\text { Folga } \\
\text { Rede }\end{array}$ \\
\hline (ERB 01, CCC 01) & 0 & 0 & 0 \\
\hline (ERB 02, CCC 01) & 0 & 0 & 0 \\
\hline (ERB 03, CCC 01) & 0 & 0 & 0 \\
\hline (ERB 04, CCC 01) & 0 & 0 & 0 \\
\hline (ERB 05, CCC 01) & 0 & 0 & 0 \\
\hline (ERB 06, CCC 01) & 0 & 0 & 0 \\
\hline (ERB 01, ERB 02) & 0 & 0 & 0 \\
\hline (ERB 01, ERB 06) & 0 & 0 & 0 \\
\hline (ERB 02, ERB 01) & 0 & 0 & 0 \\
\hline (ERB 02, ERB 03) & 0 & 0 & 0 \\
\hline (ERB 03, ERB 02) & 0 & 0 & 0 \\
\hline (ERB 03, ERB 04) & 0 & 0 & 0 \\
\hline (ERB 04, ERB 03) & 0 & 0 & 0 \\
\hline (ERB 04, ERB 05) & 0 & 0 & 0 \\
\hline (ERB 05, ERB 04) & 0 & 0 & 0 \\
\hline (ERB 05, ERB 06) & 0 & 0 & 0 \\
\hline (ERB 06, ERB 01) & 0 & 0 & 0 \\
\hline (ERB 06, ERB 05) & 0 & 0 & 0 \\
\hline
\end{tabular}

Fonte: elaborado pelos autores (2018)

Função Objetivo: refere-se ao mínimo custo do aluguel da rede de interconexão SDH. É calculada somando-se os custos de aluguel dos links escolhidos para o escoamento da demanda dos nós de ERB até o nó de CCC, conforme expressão (5), e implementa a defuzzificação do parâmetro custo de aluguel considerando a função "valor esperado" para um número fuzzy trapezoidal na expressão (6).

$$
\begin{aligned}
& \operatorname{Min} \sum_{[i] \in A_{m}} \sum_{[n]] \in O_{S D H}}\left(C_{n}+r \cdot l_{i j}\right) \cdot X_{i n} \\
& \operatorname{Min} \sum_{[i] \in A_{n}[n] \in O_{S n H}}\left(\frac{C_{n a}+C_{n b}+C_{n e}+C_{n d}}{4}+r . l_{i j}\right) \cdot X_{i n}
\end{aligned}
$$


Onde:

OSDH: conjunto de capacidades dos links SDH candidatos no arco $[i, j]{ }^{\epsilon} \mathrm{Ae}$;

Cn: custo de aluguel do sistema de transmissão SDH de capacidade [n] ${ }^{\epsilon}$ $\mathrm{OSDH}$;

lij: comprimento do arco [i, j] ${ }^{\epsilon}$ Ae;

r: custo de aluguel de rede óptica (por $\mathrm{km}$ );

Xijn: variável binária que representa a escolha do link SDH de capacidade [n] candidato no arco $[i, j]{ }^{E}$ Ae.

$\mathrm{Na}$ Tabela 6 são indicadas as células da planilha utilizadas para computar os custos parciais, quantidade de links utilizados de cada modularidade $\mathrm{SDH}$, a folga total da rede escolhida, o intervalo entre a demanda mínima e máxima e principalmente o custo total.

Tabela 6 - Cálculo da função objetivo: minimização de custo de aluguel

\begin{tabular}{|c|c|c|c|c|c|c|c|c|c|c|c|c|c|c|c|c|c|c|c|c|c|c|}
\hline \multirow{3}{*}{$\begin{array}{c}\text { Nó de } \\
\text { Origem } \\
\text { [i] }\end{array}$} & \multirow{3}{*}{$\begin{array}{c}\text { Capacidade } \\
\text { Link SDH }\end{array}$} & \multicolumn{21}{|c|}{ Nó de Destino [j] - Custo de cada Link Escolhido pelo Modelo } \\
\hline & & \multicolumn{3}{|c|}{ CCC 01} & \multicolumn{3}{|c|}{ ERB 01} & \multicolumn{3}{|c|}{ ERB 02} & \multicolumn{3}{|c|}{ ERB 03} & \multicolumn{3}{|c|}{ ERB 04} & \multicolumn{3}{|c|}{ ERB 05} & \multicolumn{3}{|c|}{ ERB 06} \\
\hline & & 21 & 42 & 63 & 21 & 42 & 63 & 21 & 42 & 63 & 21 & 42 & 63 & 21 & 42 & 63 & 21 & 42 & 63 & 21 & 42 & 63 \\
\hline & $\begin{array}{l}\text { Custo Link } \\
\text { SDH }\end{array}$ & 1,0 & 1,7 & 2,5 & 1,0 & 1,7 & 2,5 & 1,0 & 1,7 & 2,5 & 1,0 & 1,7 & 2,5 & 1,0 & 1,7 & 2,5 & 1,0 & 1,7 & 2,5 & 1,0 & 1,7 & 2,5 \\
\hline $\begin{array}{c}\text { ERB } \\
01\end{array}$ & & 0 & 0 & 0 & & & & 0 & 0 & 0 & & & & & & & & & & 0 & 0 & 0 \\
\hline $\begin{array}{c}\text { ERB } \\
02\end{array}$ & & 0 & 0 & 0 & 0 & 0 & 0 & & & & 0 & 0 & 0 & & & & & & & & & \\
\hline $\begin{array}{c}\text { ERB } \\
03\end{array}$ & & 0 & 0 & 0 & & & & 0 & 0 & 0 & & & & 0 & 0 & 0 & & & & & & \\
\hline $\begin{array}{c}\text { ERB } \\
04\end{array}$ & & 0 & 0 & 0 & & & & & & & 00 & 00 & 00 & & & & 0 & 0 & 0 & & & \\
\hline $\begin{array}{c}\text { ERB } \\
05\end{array}$ & & 0 & 0 & 0 & & & & & & & & & & 0 & 0 & 0 & & & & 0 & 0 & 0 \\
\hline $\begin{array}{c}\text { ERB } \\
06\end{array}$ & & 0 & 0 & 0 & 0 & 0 & 0 & & & & & & & & & & 0 & 0 & 0 & & & \\
\hline Funç & ão Objetivo: & & & 00 & & & & & & & & & & & & & & & & & & \\
\hline Folg & a Rede (E1): & & & 0 & & & & & & & & ema & inda & aten & ndid & & & 0 & & & & \\
\hline
\end{tabular}

Fonte: elaborado pelos autores (2018)

Deve-se destacar, uma vez que o modelo matemático é utilizado com o objetivo de minimizar os custos com o aluguel de rede, o fator (ou grau) de confiança, $\alpha=0$, representa o maior intervalo no número fuzzy, isto é, menor 
confiança. À medida que o fator de confiança, a, aproxima-se de 1, o intervalo de demanda previsto diminui. Com $\alpha=1$, o termo independente dos conjuntos de restrições (2) e (3) alcançam o mesmo valor, VM (Figura 5).

\section{RESULTADOS}

Como pode ser observado anteriormente na Figura 4, o estudo é feito para uma rede composta por 6 ERBs que necessitam de conexão com uma CCC. As ERBs podem ser interconectadas com a CCC através de 6 arcos diretos ou por 12 arcos indiretos entre as ERBs.

Os cenários escolhidos para a realização das simulações computacionais têm como objetivo avaliar o comportamento da rede a ser alugada conforme o grau de confiança (parâmetro $\alpha$ ) do planejador nos dados de custo de aluguel de rede, Quadro 1, e demanda imprecisa, conforme o Quadro 2. O custo de aluguel por quilometro da rede ótica é admitido como conhecido e constante, valor de $0,10 \$ / \mathrm{km}$.

Quadro 1 - Capacidade e custo impreciso de aluguel para os links SDH candidatos

\begin{tabular}{|c|c|c|c|c|c|c|}
\multicolumn{1}{c|}{} & \multicolumn{5}{c|}{ Custos $(\$)$} & \multirow{2}{*}{$\begin{array}{c}\text { Valor } \\
\text { Lédio }\end{array}$} \\
\cline { 2 - 7 } \multicolumn{2}{c|}{ Links - Cap } & a & b & c & d & 1,00 \\
\hline link 21 & $\tilde{C} 21$ & 0,90 & 0,95 & 1,05 & 1,10 & 1,70 \\
\hline link 42 & $\tilde{C} 42$ & 1,53 & 1,62 & 1,79 & 1,87 & 1,70 \\
\hline link 63 & $\tilde{C} 63$ & 2,25 & 2,38 & 2,63 & 2,75 & 2,50 \\
\hline
\end{tabular}

Fonte: elaborado pelos autores (2018)

Com a variação do fator de confiança $\alpha$ é possível abranger a imprecisão dos dados de demanda e do preço de aluguel do sistema $\mathrm{SDH}$, tornando assim mais próximo a realidade do mercado. Observa-se o valor da função objetivo (de mínimo custo), as restrições que o modelo de otimização deve atender com destaque para a garantia de atendimento de demanda mínima e a garantia de capacidade técnica dos links SDH. 
Quadro 2 - Demanda imprecisa prevista para cada nó de ERB

\begin{tabular}{|c|c|c|c|c|c|c|c|}
\hline \multirow{2}{*}{ ERBs } & \multicolumn{4}{|c|}{ Demanda em canais E1 } & \multirow{2}{*}{$\begin{array}{l}\text { Limite } \\
\text { inferior }\end{array}$} & \multirow{2}{*}{$\begin{array}{l}\text { Limite } \\
\text { superior }\end{array}$} & \multirow{2}{*}{$\begin{array}{c}\text { VM } \\
\text { demanda }\end{array}$} \\
\hline & A & $b$ & c & $d$ & & & \\
\hline D̃1 & 39,6 & 41,8 & 46,2 & 48,4 & 40 & 48 & 44 \\
\hline D̃2 & 27 & 28,5 & 31,5 & 33 & 27 & 33 & 30 \\
\hline D̃3 & 34,2 & 36,1 & 39,9 & 41,8 & 35 & 41 & 38 \\
\hline D̃4 & 12,6 & 13,3 & 14,7 & 15,4 & 12 & 16 & 14 \\
\hline D̃5 & 30,6 & 32,3 & 35,7 & 37,4 & 31 & 37 & 34 \\
\hline D̃6 & 18 & 19 & 21 & 22 & 18 & 22 & 20 \\
\hline
\end{tabular}

Fonte: elaborado pelos autores (2018)

A rede foi avaliada inicialmente para valores atuais de demanda e de custo de aluguel de rede óptica trabalhados em Oliveira e DeSousa (2015). Na sequência, foram feitas simulações com aplicação fuzzy trapezoidal. A Tabela 7 mostra os resultados obtidos de acordo com o grau de confiança nos dados de demanda. São indicados: parâmetro $\alpha$, intervalo em que a demanda pode variar, a demanda atendida, custo total, a folga total e a quantidade de links utilizados em cada rede escolhida.

Por ser um problema de minimização, e devido à forma como foram modeladas as restrições de balanço de demanda, a curva de defuzzyficação acaba por retornar o menor valor do intervalo fuzzy em análise, limitante inferior. Pode ser observado que, para a estrutura de custos adotada, a topologia da rede escolhida se mantém constante até aproximar do maior grau de confiança a e consequentemente o do custo total da rede. Destacam-se duas topologias de rede, uma para o grau de confiança entre $0 \%$ e $80 \%$, com custo de $\$ 9,97$, e outra para o intervalo entre $90 \%$ e $100 \%$, com custo de $\$ 10,77 \$$. A medida que o grau de confiança aumenta, cresce a demanda prevista para ser atendida, como a topologia da rede se mantêm-se, observa-se que a folga total da rede vai diminuindo até atingir o limiar de 39xE1. A partir daí, acontece a troca de um enlace de 42xE1 por outro de 63xE1. 
Tabela 7 - Resultados da simulação

\begin{tabular}{|c|c|c|c|c|c|c|c|c|}
\hline$\alpha$ & $\begin{array}{l}\text { Inter } \\
d \\
\text { dem }\end{array}$ & & $\begin{array}{l}\text { Demanda } \\
\text { atendida }\end{array}$ & Custo total & $\begin{array}{c}\text { Folga } \\
\text { Rede (E1): }\end{array}$ & Link21 & Link42 & Link63 \\
\hline 0,0 & 150 & 210 & 150 & 9,97 & 60 & 2 & 4 & 0 \\
\hline 0,1 & 153 & 207 & 153 & 9,97 & 57 & 2 & 4 & 0 \\
\hline 0,2 & 155 & 205 & 155 & 9,97 & 55 & 2 & 4 & 0 \\
\hline 0,3 & 158 & 202 & 158 & 9,97 & 52 & 2 & 4 & 0 \\
\hline 0,4 & 161 & 199 & 161 & 9,97 & 49 & 2 & 4 & 0 \\
\hline 0,5 & 166 & 197 & 163 & 9,97 & 47 & 2 & 4 & 0 \\
\hline 0,6 & 170 & 194 & 166 & 9,97 & 44 & 2 & 4 & 0 \\
\hline 0,7 & 171 & 190 & 170 & 9,97 & 40 & 2 & 4 & 0 \\
\hline 0,8 & 174 & 189 & 171 & 9,97 & 39 & 2 & 4 & 0 \\
\hline 0,9 & 18 & 186 & 174 & 10,77 & 57 & 2 & 3 & 1 \\
\hline 1,0 & 180 & 180 & 180 & 10,77 & 51 & 2 & 3 & 1 \\
\hline
\end{tabular}

$\overline{\text { Fonte: }}$ elaborado pelos autores (2018)

A topologia de Rede 1 (com a entre 0 e 80\%) é mostrada na Figura 6 e apresenta o cenário de atendimento com $0 \%$ de confiança na previsão de demanda. São indicados a demanda escoada em cada arco escolhido, bem como a capacidade (modularidade) do sistema de transmissão SDH alugado.

A Rede 2 (com a entre 90\% e 100\%) apresenta a topologia para o cenário de atendimento com $100 \%$ de confiança na previsão de demanda em que são indicados a demanda escoada em cada arco escolhido, bem como a capacidade (modularidade) do sistema de transmissão SDH alugado (Figura 7). Os resultados mostram que as soluções de rede privilegiaram os links diretos entre ERB - CCC. 
Figura 6 - Topologia com $0 \%$ de confiança de demanda [37]

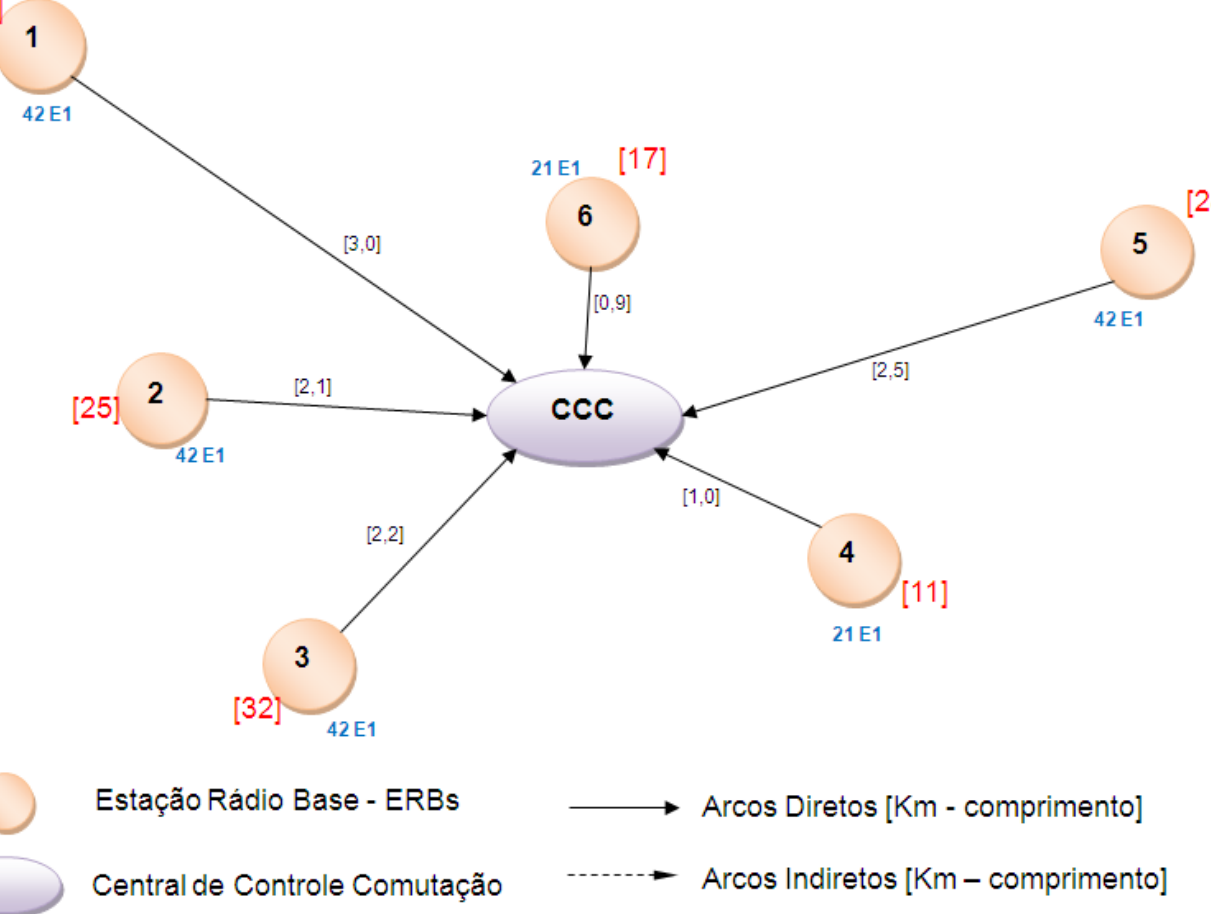

Fonte: elaborado pelos autores (2018)

Figura 7 - Topologia com 100\% de confiança de demanda

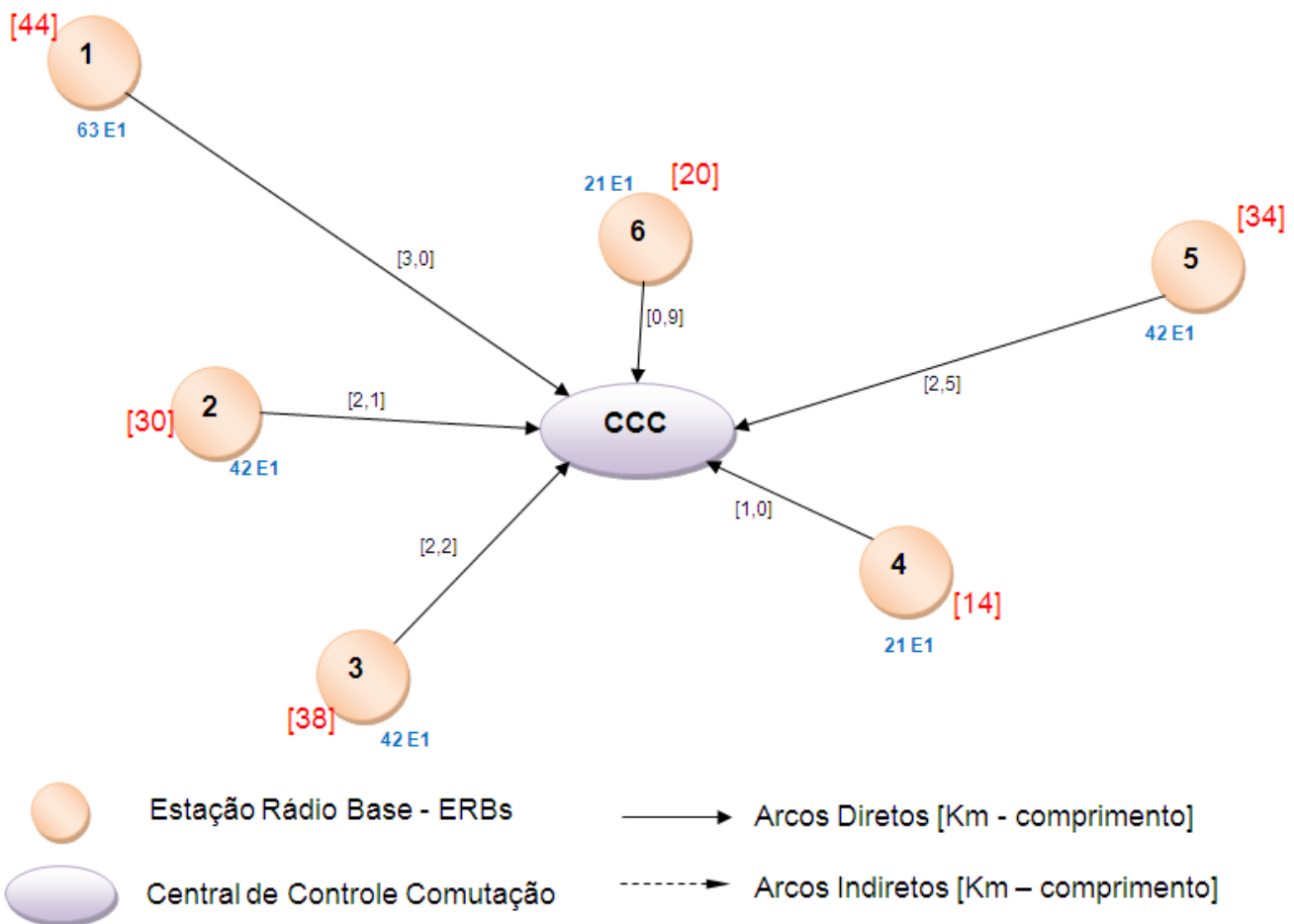

Fonte: elaborado pelos autores (2018) 


\section{DISCUSSÃO}

Os cenários gerados a partir da variação do fator de confiança têm por objetivo avaliar o comportamento da rede a ser alugada e verificar se a imprecisão nos dados pode gerar mudanças significativas na topologia da rede. Como mostrado anteriormente, na figura 7 , ao variar o fator $\alpha$, para a primeira topologia percebe-se que a demanda atendida é inversamente proporcional a folga da rede. E a segunda, ocorre por causa do aumento da demanda que consequentemente requer de determinados enlaces uma maior capacidade em canais E1.

No cenário 1 , com uma previsão de até $80 \%$ nas demandas previstas, a simulação configurou o sistema de forma a obedecer a todos os parâmetros estabelecidos e escolheu em todo intervalo de variação do $\alpha$, a melhor solução de custo total de aluguel da rede de $\$ 9,97$, com uma folga em canais $E 1$ inversamente proporcional a demanda atendida. Foram escolhidos 2 arcos com capacidade de $21 x E 1$ e 4 arcos com capacidade de 42xE1. A topologia da rede escolhida pelo Sistema de Apoio à Decisão em relação ao dimensionamento dos enlaces resultou no escoamento através de arcos que ligam as ERBs diretamente com a CCC.

No cenário 2, com maior precisão nas demandas previstas, com alfa entre $90 \%$ e $100 \%$, a simulação configurou a melhor solução, com custo total de aluguel da rede de $\$ 10,77$, com folga em canais $E 1$ inversamente proporcional a demanda atendida e maior devido a alocação dos arcos. Foram escolhidos 2 arcos com capacidade de 21xE1, 3 arcos com capacidade de 42xE1 e 1 arco com capacidade de $63 \times E 1$. A topologia da rede escolhida privilegiou o escoamento de demanda por arcos diretos a CCC.

$\mathrm{Na}$ simulação computacional foi utilizado o Solver que é um suplemento do software da planilha eletrônica (Microsoft Office Excel), sendo executado no sistema operacional Windows XP, com processador Intel Centrino de $1,66 \mathrm{GHz}$ de velocidade e $2 \mathrm{~GB}$ de memória RAM. O tempo computacional, para todos os cenários avaliados, consumiu em torno de 3 minutos de processamento. Vale lembrar que o problema avaliado neste trabalho possui em torno de 70 variáveis, sendo 54 variáveis binárias. Por não ser um software exclusivo de modelagem para otimização, o aumento da rede, e consequentemente da quantidade de variáveis 
pode aumentar exponencialmente o tempo de processamento do modelo pelo algoritmo utilizado.

\section{CONCLUSÃO}

O constante desenvolvimento do mercado de comunicação móvel celular tem obrigado as operadoras do setor a buscarem soluções novas de planejar suas redes, aliando tanto a minimização de custos quanto a maximização das receitas. Ao avaliar as mais variadas possibilidades de capacidade de atendimento da infraestrutura de interconexão de ERBs em um sistema de comunicação móvel, destaca-se a possibilidade de classificação de redes, permitindo ao planejador selecionar aquela que atende as suas exigências técnicas. A possibilidade de aluguel de rede é uma alternativa que também deve ser avaliada pela operadora do sistema em constante atualização.

Este trabalho apresentou uma proposta de planejamento estratégico orientado à minimização de custos de aluguel para a infraestrutura de interconexão de ERBs em um Sistema Móvel Celular através do dimensionamento de links de transmissão $\mathrm{SDH}$. A rede é vista como um grafo e a modelagem é traduzida como um problema de programação linear inteira mista, o qual obedece a restrições técnicas de demanda e capacidade seguindo a abordagem NÓ - ARCO. Os dados imprecisos de demanda e custo foram modelados como números fuzzy trapezoidais. Como a CCC não tem restrição de capacidade técnica, ou seja, ela é capaz de absorver toda demanda existente e sua possível variação de aumento ou decremento, sugere-se aprofundar o modelo de forma a respeitar a capacidade real do equipamento e a utilização de n CCCs, utilizando uma abordagem ARCO CAMINHO, de forma a atender o mercado utilizando uma ferramenta como o Excel.

O modelo matemático do Sistema de Apoio à Decisão utilizado nesta pesquisa demonstrou ser um facilitador para o planejador avaliar como é o comportamento da rede para as mais variadas possibilidades. Mostrou maior eficácia do que o proposto em Oliveira e DeSousa (2015), uma vez que os esforços computacionais necessitaram de menos tempo de processamento computacional para a obtenção das soluções, além de permitir uma modelagem mais realística. A utilização do solver na planilha eletrônica, para resolver problemas de programação 
linear inteira mista, permitiu aprimorar o uso de uma ferramenta computacional de fácil utilização por parte do usuário final do sistema, de forma a facilitar o treinamento de equipes em planejamento estratégico, em redes de comunicação móvel celular e modelagem matemática utilizando pesquisa operacional.

Enfim, a ferramenta computacional facilita o processo de decisão durante o dimensionamento e a avaliação de risco no planejamento, mostrando-se muito promissora. Como não é uma ferramenta exclusiva, sugere-se a utilização da planilha eletrônica junto à outra ferramenta de simulação e modelagem para melhorar o tempo computacional e resultados obtidos. Cenários com um número maior de nós de ERB e técnicas mais apuradas para o tratamento da imprecisão na função objetivo são os desafios indicados como trabalhos futuros.

\section{AGRADECIMENTO}

Os autores agradecem Coordenação de Aperfeiçoamento de Pessoal de Nível Superior (CAPES) que contribuiu para a elaboração deste trabalho.

\section{REFERÊNCIAS}

AKASAKA, Y; D.'AGOSTO, M. A.; RIBEIRO, G. M. Modelo matemático para estruturação do sistema logístico reverso do resíduo sólido urbano para coprocessamento. Revista Produção Online, v. 16, n. 3, pp. 844-874, 2016. DOI: https://doi.org/10.14488/16761901.v16i3.2131

ALLON, G.; FEDERGRUEN, A.. Competition in service industries. Operations Research. V. 55, N. 1, pp. 37-55, 2007. DOI: http://dx.doi.org/10.1287/opre.1060.0337

ANATEL. Serviço móvel celular. Disponível em: http://www.anatel.gov.br/portal/exibirPortallnternet.do. Acesso em: 26 de nov. 2014.

ANDERSON, E.; KELLY, F.; STEINBERG, R.. A contract and balancing mechanism for sharing capacity in a communication network. Management Science, v. 52, n. 1, p. 39-53, 2006.

https://www.researchgate.net/publication/227447640 A Contract and Balancing Mechanis $\mathrm{m}$ for Sharing Capacity in a Communication Network

Asga. Especificações técnicas de modems ópticos. Disponível em: http://www.asga.com.br/category/produtos/. Acesso em: 26 de nov. 2014.

CAMPOS, E.S.; NOGUEIRA, M.C.; SOUZA, F.J.C.; DeSOUSA, M.A. Um Modelo MILP 0-1 para o Dimensionamento de Rede SDH de Longa Distância em um SMC. ERPO-NE, 4., 2013. Anais... Salvador-BA. 2013. 
DE CASTRO, D.; REIS, J. G. M.; VENDRAMETTO, O.; LOPES, A. C. V. Modelo de decisão multicritério para escolha do modo de transporte: um estudo do escoamento da produção de grãos de Mato Grosso do Sul. Revista Produção Online, v. 16, n. 4, pp. 1214-1236, 2016. DOI: https://doi.org/10.14488/1676-1901.v16i4.2206

DEFIGUEIREDO, R. M.; CANTODOSSANTOS, J.V. Um comparativo entre métodos computacionais para planejamento de redes de telecomunicações. Revista Brasileira de Computação Aplicada, v. 5, n. 1, pp. 14-25, 2013.DOI:

http://dx.doi.org/10.5335/rbca.2013.2579

DE SOUSA, M. A; CARLSON, C. M.F; VIEIRA, F. H. T. Optimum Allocation of Transmission Technologies for Solving the BTS Interconnection Problem in Cellular Systems.

In: Handbook of research on artificial intelligence techniques and algorithms. IGI Global, pp. 152-182, 2015.

DESOUSA, M. A.; SOUZA, F. J. C.; VIERIRA, F. H. T.; COSTA, V. H. T.; CAMPOS, E. S.. Interconexão de ERBs em um SMC - Um MILP para alocação e dimensionamento de enlaces SDH. SIMPÓSIO BRASILEIRO DE TELECOMUNICAÇÕES - SBRT2013, 31., 2013, Fortaleza- CE. Anais ... Rio de Janeiro - RJ: Sociedade Brasileira de Telecomunicações SBrT, 2013. pp.1-5, 2013.

DIGITEL. especificações técnicas de modems SHDSL. Disponível em: http://www.digitel.com.br/pt/produtos/. Acesso em: 10 de nov. 2015.

ERICSSON. Especificações técnicas de sistemas de rádio microondas. Disponível em: < https://www.ericsson.com/ourportfolio/products/microwave-networks >. Acesso em: $10 \mathrm{de}$ Novembro de 2015.

GARG, V. K..Wireless communications and networking. 1. ed. Rio de Janeiro: Elsevier, 2007. 840 p.

GEORGE, J.K.; BO, Y.. Fuzzy sets and fuzzy logic, theory and applications, 1. ed. Prentice Hall, 1995. 592 p.

INGA, E.; AREVALO, G.; HINCAPIÉ, R. Optimal deployment of cellular networks for Advanced Measurement Infrastructure in Smart Grid. In: Communications and Computing (COLCOM), 2014 IEEE Colombian Conference on. IEEE. pp. 1-6, 2014.

DOI: htp://dx.doi.org/10.1109/ColComCon.2014.6860421

JESZENSKY, P. J. E. Sistemas telefônicos. 1. ed. São Paulo: Manole, 2004. 651p.

KASAP, N.; AYTUG, H.; ERENGUC, S.S.. Provider selection and task allocation issues in networks with different QoS levels and all you can send pricing. Decision Support Systems, v. 43, n. 2, pp. 375-389, 2007. DOI: http://dx.doi.org/10.1016/i.dss.2006.10.008

LETOURNEUX, F.; CORRE, Y.; SUTEAU, E.; LOSTANLEN, Y.. 3D coverage analysis of LTE urban heterogeneous networks with dense femtocell deployments. EURASIP Journal on Wireless Communications and Networking, 2012.

http://jwcn.eurasipjournals.springeropen.com/articles/10.1186/1687-1499-2012-319

MADRONERO, D. M.; MULA, J.; JIMÉNEZ, M.. A modified approach based on ranking fuzzy numbers for fuzzy integer programming with equality constraints. 6th International

Conference on Industrial Engineering and Industrial Management. Vigo, 2012. 
http://www.adingor.es/congresos/web/uploads/cio/cio2012/EN 03 Production and Operatio ns Management/375-382.pdf

MATHUR, N.; SRIVASTAVA, P. K.; PAUL, A. Trapezoidal fuzzy model to optimize transportation problem. International Journal of Modeling, Simulation, and Scientific Computing, v. 7, n. 03, p. 1650028, 2016. DOI:

https://doi.org/10.1142/S1793962316500288

NIYATO, D.; HOSSAIN, E.. A queuing-theoretic and optimization-based model for radio resource management in IEEE 802.16 broadband wireless networks. IEEE Transactions on Computer, v. 55, n. 11, pp.1473-1488, 2006. DOI: http://dl.acm.org/citation.cfm?id=1176136

OLIVEIRA, L.S.; DeSOUSA, M.A.. Sistema de apoio à decisão via planilha eletrônica para o problema de interconexão de ERBs em um sistema móvel celular utilizando a tecnologia SDH. XXXV Encontro Nacional de Engenharia de Produção. Fortaleza-CE. 2015. http://www.abepro.org.br/biblioteca/TN STO 211253 27454.pdf

OLIVEIRA, B. Q. Modelos de otimização plim-fuzzy para o dimensionamento de rede e seleção de serviços em telecomunicações. Dissertação apresentada ao Programa de Mestrado em Engenharia de Produção e Sistemas - MEPROS, PUC Goiás, 2015.

PEDRYCZ, W.; GOMIDE, F. An Introduction to fuzzy sets: analysis and design. 1. Ed. MIT Press, Cambridge, MA, 1998. 490 p.

PEIDRO, D.; MULA, J.; JIMÉNEZ, M.; MAR BOTELLA, M.. A fuzzy linear programmingbased approach for tactical supply chain planning in an uncertainty environment. European Journal of Operational Research, v. 205, n. 1, p. 65-80, 2010.

DOI: http://dx.doi.org/10.1016/j.ejor.2009.11.031

ROUSKAS, A. N.; KIKILIS, A.A.; RATSIATOS, S.S.. A game theoretical formulation of integrated admission control and pricing in wireless networks. European Journal of Operational Research, v. 191, n. 3, p.1175-1188, 2008.

DOI: http://dx.doi.org/10.1016/..ejor.2007.07.017

SAM'AN, M.; Farikhin; HARIYANTO, S.; SURARSO, B. Optimal solution of full fuzzy transportation problems using total integral ranking. Journal of Physics: Conference Series. IOP Publishing, 2018. p. 012075.

https://doi.org/10.1088/1742-6596/983/1/012075

SKIANIS, C.. Introducing automated procedures in $3 G$ network planning and optimization. Journal of Systems and Software, v. 86, n. 6, pp. 1596-1602, 2013.

DOI: http://dx.doi.org/10.1016/i.jss.2013.01.072

TELECO. Tutoriais sobre sistemas de comunicação móvel celular e comunicação óptica. Disponível em: http://www.teleco.com.br/tutoriais.asp. Acesso em: 23 out. 2014.

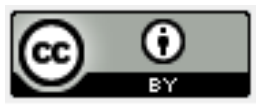

Artigo recebido em: 03/03/2017 e aceito para publicação em: 09/11/2018 DOI: http://dx.doi.org/10.14488/1676-1901.v18i4.2763 